\title{
AUTOPERCEPÇÃO DO PRÉ-NATAL ODONTOLÓGICO PELAS GESTANTES DE UMA UNIDADE BÁSICA DE SAÚDE
}

\section{Autoperception of dentistry prenatal by the pregnant women of a Basic Health Unit}

Ingrid Karem Rangel Lopes - Cirurgiã-Dentista Residente do Programa de Residência Multiprofissional em Atenção Básica - Escola Multicampi de Ciências Médicas (EMCM) da Universidade Federal do Rio Grande do Norte (UFRN). E-mail: ingridkarem@hotmail.com

Daniela Mendes da Veiga Pessoa - Mestre em Odontologia Preventiva e Social pela UFRN. Doutora em Ciências da Saúde pela (UFRN). Professora Adjunto IV do Curso de Odontologia da Universidade Estadual do Rio Grande do Norte (UERN). E-mail: danielamvp@yahoo.com.br

Giulian Lennon de Macêdo - Cirurgião-Dentista Residente do Programa de Residência Multiprofissional em Atenção Básica - Escola Multicampi de Ciências Médicas (EMCM) da Universidade Federal do Rio Grande do Norte (UFRN). E-mail: giulian.lennon@gmail.com

Autor responsável pela correspondência:

Ingrid Karem Rangel Lopes.

E-mail: ingridkarem@hotmail.com 


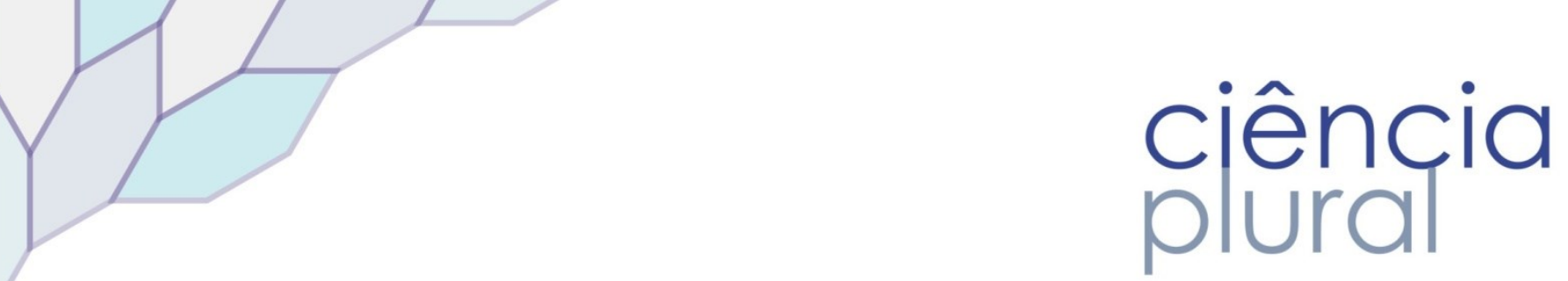

\section{RESUMO}

Introdução: O tratamento odontológico durante o pré-natal desencadeia medo nas mães, visto que ainda circunda o mito de que seria prejudicial à saúde do bebê e da gestante, proporcionando o distanciamento da gestante à atenção odontológica. Objetivo: Identificar autopercepção das gestantes sobre pré-natal odontológico. Métodologia: Pesquisa de natureza qualitativa, do tipo exploratória. Foi aplicado um instrumento com questões abertas, contendo dados socioeconômicos e informações sobre a percepção do pré-natal odontológico pelas gestantes usuárias dos serviços de uma Unidade Básica de Saúde do interior do Rio Grande do Norte. Para análise dos dados foi realizada a triangulação dos resultados obtidos pelas técnicas de coleta: entrevista, análise documental e teoria científica. Resultados: $83,3 \%$ das gestantes ouviram falar em pré-natal odontológico, $75 \%$ delas afirmaram que foram orientadas por algum profissional de saúde sobre a importância de se fazer o pré-natal odontológico, onde os enfermeiros foram os mais citados. Todas as gestantes afirmaram ser importante ir a uma consulta odontológica durante a gestação, $91,7 \%$ das usuárias foram a uma consulta odontológica durante a gravidez e 91,7\% acreditam que alguma alteração na boca durante a gestação pode interferir na saúde geral do bebê. Conclusão: As gestantes consideram importante o pré-natal odontológico, pois veem como uma oportunidade de acesso aos serviços de saúde, compareceram a consulta odontológica e apresentaram um grau de adesão ao aconselhamento fornecido pelos profissionais de saúde.

Palavras-chave: Atenção odontológica; Gestantes; Percepção; Saúde bucal; Pré-natal.

\section{ABSTRACT}

Introduction: Dental treatment during prenatal care triggers fear in mothers, since it still surrounds the myth that it would be harmful to the health of the baby and the pregnant woman, thus distancing the pregnant woman to dental care. Objective To identify self-perception of pregnant women about dental prenatal care. Methodology: Research of a qualitative nature, exploratory type. An instrument with open questions, containing socioeconomic data and information about the perception of prenatal dentistry by pregnant women users of the services of a Basic Health Unit in the interior of Rio Grande do Norte, was applied. For the analysis of the data, the triangulation of the results obtained by the collection techniques was performed: interview, documentary analysis and scientific theory. Results: $83.3 \%$ of the pregnant women heard about prenatal dentistry, $75 \%$ of them said that they were advised by a health professional about the importance of doing prenatal dentistry, where nurses were the most cited. All the pregnant women stated that it was important to go to a dental appointment during pregnancy, $91.7 \%$ of the users went to a dental appointment during pregnancy and $91.7 \%$ believed that any changes in the mouth during pregnancy could interfere with their overall baby. Conclusion: Pregnant women consider dental prenatal to be important, since they see as an opportunity to access health services, attended the dental consultation and presented a degree of adherence to the advice provided by health professionals.

Keywords: Dental Care; Pregnant women; Perception; Oral health; Prenatal. 


\section{ciência \\ plural}

\section{Introdução}

O objetivo do acompanhamento pré-natal é assegurar o desenvolvimento da gestação, permitindo 0 parto de um recém-nascido saudável, sem impacto para a saúde materna, inclusive com a abordagem dos aspectos psicossociais e das atividades educativas e preventivas. Os profissionais de saúde devem realizar ações de atenção integral e de promoção de saúde, prevenção de agravos e escuta qualificada das necessidades dos usuários em todas as ações, proporcionando atendimento humanizado e viabilizando 0 estabelecimento do vínculo. É importante também, realizar a busca ativa e a notificação de doenças e agravos. $^{1}$

Em relação à saúde bucal das gestantes, observa-se que algumas manifestações são mais comuns durante a gestação como, por exemplo, a cárie e a doença periodontal, embora a gravidez não seja a principal responsável por tais afecções. 0 aparecimento da cárie está relacionado ao alto índice de Estreptocous Mutans, deficiência no controle do biofilme dentário, alto consumo de açúcares e acesso reduzido aos serviços de saúde. ${ }^{2}$ Já a doença periodontal tem o biofilme como principal fator etiológico e deve ser vista como um processo de desequilíbrio entre as ações de agressão e defesa sobre os tecidos periodontais, que é modificada de acordo com as respostas dadas pelo hospedeiro. Na gestante, as alterações da composição do biofilme subgengival, a resposta imunológica e a concentração de hormônios sexuais são fatores que influenciam a resposta do periodonto. ${ }^{3}$

Desta forma, faz-se necessário acompanhamento das gestantes no pré-natal odontológico. Este é visto como parte relevante dos cuidados durante a gravidez, devido aos aspectos biológicos, clínicos e pelo compartilhamento de fatores culturais, comportamentais e socioeconômicos do ambiente familiar. ${ }^{4}$

Em relação aos mitos, no passado, cirurgiões-dentistas eram advertidos a tratarem gestantes somente durante o segundo trimestre gestacional ou em casos de urgências. Dessa forma, até os dias atuais, muitos profissionais sentem-se inseguros para prestação de serviço pré-natal e, na maioria das vezes, postergam os atendimentos para a fase pós-parto. ${ }^{5}$ Porém, a maioria dos procedimentos odontológicos podem ser realizados, em qualquer período gestacional, tendo alguns cuidados, como sessões mais curtas e posições adequadas na cadeira. A gestação com um bom prognóstico, cujo tratamento envolve apenas a prevenção, profilaxia e restaurações e tratamento periodontal simples devem ser tratadas na Unidade Básica de Saúde (UBS), ao longo da gestação, pois não oferecem danos a gestante nem para o bebê. ${ }^{6}$ 


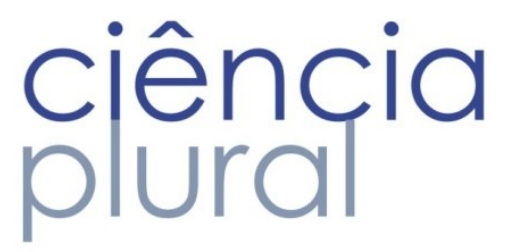

Contudo, o tratamento odontológico durante o pré-natal desencadeia medo nas mães, visto que, ainda circunda o mito de que seria prejudicial à saúde do bebê e da gestante, proporcionando o distanciamento da gestante à atenção odontológica. ${ }^{7}$ Desta forma, a gravidez não deve ser um motivo para postergar 0 tratamento odontológico, pois a gestante encontra-se psicologicamente receptiva a adquirir novos conhecimentos. $^{8}$

Diante do exposto, pode-se usar esse momento de receptividade de informações por parte das gestantes e desenvolver ações educativo-preventivas para empoderar as usuárias sobre a gestação e 0 autocuidado e através da adesão ao aconselhamento proporcionar benefícios para a mãe e filho. ${ }^{9}$

Diante do exposto, fez-se necessária a realização do presente estudo, pioneiro no município de Currais Novos, localizado na região Seridó do Rio Grande do Norte (RN), para identificar a percepção das gestantes usuárias dos serviços públicos sobre o pré-natal odontológico e consequentemente contribuir para um maior entendimento por parte dos profissionais das equipes de saúde bucal e dos demais profissionais diretamente relacionados ao cuidado às gestantes, no intuito do desenvolvimento de ações de promoção da saúde bucal voltadas para esta população, a partir de suas necessidades e percepção acerca do pré-natal odontológico.

\section{Metodologia}

O presente estudo, de natureza qualitativa, do tipo exploratório, foi realizado na Unidade Básica de Saúde (UBS) Joaninha Parteira existente há 15 anos no município de Currais Novos, localizado na região Seridó do Rio Grande do Norte (RN). Esta cidade, segundo IBGE possui uma população de 45.060 habitantes e é considerado município polo na região por obter serviços de referência para Currais Novos e demais municípios da região do Seridó. Atualmente apresenta com 17 Equipes Saúde da Família (ESF) e 15 Equipes de Saúde Bucal (ESB). A UBS conta com a presença dos residentes do Programa Multiprofissional em Atenção Básica EMCM/UFRN e desenvolvem junto à ESF grupos específicos voltados para as Práticas Integrativas e Complementares, acompanhamento de gestantes, hipertensos e diabéticos, mulheres menopausadas e usuários que desenvolvem a função de cuidadores de idosos acamados e domiciliados.

A escolha desta UBS para o desenvolvimento da referida pesquisa é justificada pelo fato desta ser considerada uma unidade onde existe o contraste entre a adesão por parte das gestantes ao pré-natal de risco habitual e, ao mesmo tempo, a sua falta no pré-natal odontológico. 


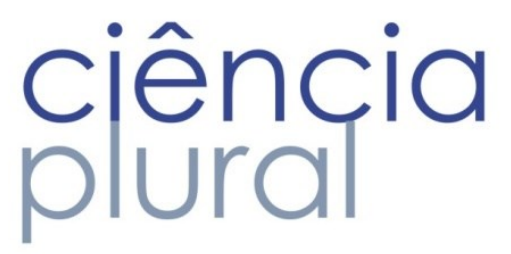

O estudo de abordagem qualitativa não tem intenção de quantificar uma realidade e sim trabalhar com um universo de motivos, crenças, valores, atitudes, correspondendo a um espaço mais profundo nas relações dos processos e dos fenômenos. ${ }^{10}$

Fizeram parte do estudo 12 gestantes (de um total de 20 mulheres grávidas acompanhadas pela UBS), tendo como critérios de inclusão, serem maior de idade, cadastradas em áreas de abrangência da unidade básica de saúde e que estavam em acompanhamento do pré-natal de risco habitual na UBS Joaninha Parteira.

Para a coleta de dados, foi realizada uma entrevista dividida em duas partes: uma que envolve variáveis socioeconômicas, tais como idade, estado civil, escolaridade, renda familiar e vínculo empregatício da gestante e a segunda parte com perguntas sobre a autopercepção da gestante sobre 0 pré-natal odontológico.

O recrutamento das participantes aconteceu com auxílio das Agentes Comunitárias de Saúde da UBS. Logo após os esclarecimentos sobre o objetivo da pesquisa, o método de coleta, seguidos da leitura, explicação e assinatura pelas gestantes do Termo de Consentimento Livre e Esclarecido (TCLE) e do Termo de Autorização para Gravação de Voz, as entrevistas foram iniciadas em um período de Novembro de 2017 a Janeiro de 2018.

As entrevistas foram realizadas por uma única entrevistadora, com auxílio de um gravador de voz, na sala de atendimento individualizado, ou em domicilio da própria entrevistada, garantindo a privacidade e sigilo das informações. A coleta de dados foi encerrada no momento em que a pesquisadora percebeu que nenhuma informação nova surgia durante o seguimento das entrevistas, sendo considerada a teoria da amostragem por saturação.

A técnica da amostragem por saturação, ferramenta empregada nas metodologias qualitativas na área da saúde, define o número de participantes do estudo a partir de quantos forem necessários para saturar os questionamentos relacionados à temática. ${ }^{11}$

As entrevistas foram transcritas na íntegra para posterior análise de seu conteúdo. Para análise dos dados foi realizada a triangulação dos resultados obtidos pelas técnicas: entrevista, análise documental e teoria cientíica. As entrevistas foram numeradas (1 a 12) de maneira aleatória e utilizada a abreviação $G$ (gestante) para diferenciar a fala de cada uma delas.

A partir do conteúdo das entrevistas e da análise dos documentos e da teoria cientifica, procurou-se obter uma resposta aos objetivos da pesquisa, buscando elucidar a percepção das gestantes acerca do pré-natal odontológico. Este estudo foi aprovado pelo Comitê de Ética da Faculdade de Ciências da Saúde do Trairi (Parecer 2.357.572). 


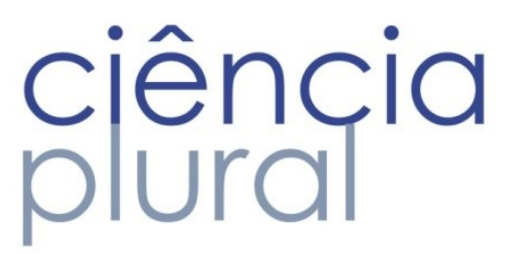

\section{Resultados e Discussão}

Em relação às características sociodemográficas das participantes da pesquisa, observou-se que das 12 gestantes, $58,4 \%$ prevaleceram dentro da faixa etária de 26 a 35 anos, 33,4\% estavam dentro da faixa etária de 15 a 25 anos e 8,4\% na faixa etária de 36 a 45 anos. De acordo com a quantidade de gestações, a metade delas relatou ser primigesta, 33,4\% disseram ser a segunda gestação, 16,7\% ser a terceira gestação. Tendo assim um perfil de mães jovens e de primeira gestação sendo características importantes da amostra, pois elas estão receptivas à novas informações referentes aos cuidados com o binômio mãefilho, possibilitando a desmistificação de crenças e preocupações sobre o tratamento odontológico.

Quanto à escolaridade, 75\% estudaram até ensino médio, 8,4\% até o ensino fundamental incompleto, $8,4 \%$ até o ensino fundamental completo, $8,4 \%$ cursando ensino superior. Quando questionadas sobre estado civil, $50 \%$ delas disseram ser casadas e $50 \%$ solteiras. Na renda mensal da família, metade relatou ganhar até dois salários mínimos, $41,7 \%$ até um salário mínimo e 8,4\% até cinco salários mínimos.

É importante ressaltar que a condição socioeconômica exerce influência na vida das pessoas, podendo refletir nos seus hábitos e comportamentos. ${ }^{12}$ Outro fator que também pode ser influenciado é a compreensão das informações compartilhadas pelos profissionais de saúde que planejarão suas ações de acordo com o nível de entendimento das usuárias.

Em alguns estudos, verificou-se que à medida que diminui a classe socioeconômica das pessoas, menor é o seu nível de conhecimento, onde mães de nível socioeconômico menos favorecido apresentam menor conhecimento em saúde bucal. ${ }^{12,13}$

Com base nos dados coletados, pode-se aventar a possibilidade de que as condições socioeconômicas, para a população pesquisada, foi um fator preditor no acesso às informações sobre saúde bucal.

Em relação à percepção sobre o pré-natal odontológico, 83,3\% das gestantes afirmaram que já tinham ouvido falar sobre o tema. Percebeu-se em algumas falas que o pré-natal odontológico é visto como uma oportunidade de acesso, evitando assim as filas exaustivas na tentativa de pegar fichas para resolução de problemas de saúde bucal pré-existentes. Também relatou-se o desconhecimento de que saúde é direito de todos e dever do estado previsto na Constituição Federal de 1988, podendo-se exemplificar a partir de algumas falas: 
"Já ouvi falar, e é assim, muito bom porque assim, a gente tem a facilidade por estar grávida na hora que procurar ser bem atendida e faz o tratamento todinho." (G 04)

"Já ouvi falar sim, é uma ajuda no tratamento odontológico, principalmente pras gestantes que tem, ficam com mais sensibilidade à cárie, doença periodontal." (G 02)

"Sim, pelo que já me explicaram é, porque a mulher no pré-natal pode surgir vários problemas bocal, como gingivite, sangramento gingival, pode chegar a estragar alguns dentes, ter algum problema, ter dor dente, é isso que eu cheguei a ver o que é." (G 07)

"Sim, na minha opinião seria muito importante pra não ter o parto prematuro." (G 09)

Em relação à orientação feita por algum profissional de saúde sob a importância de se realizar o prénatal odontológico, $75 \%$ das gestantes tiveram acesso à informação. Entre os profissionais citados pelas gestantes, os enfermeiros tiveram maior representatividade com $50 \%$, seguidos do dentista com $21,4 \%$, do Agente Comunitário de Saúde com 14,3\% e do médico e do técnico de enfermagem com 7,1\% cada.

No âmbito da saúde da mulher, especificamente tratando-se da prática obstétrica, o enfermeiro exerce um papel importante no que concerne a humanização da assistência, tendo em vista que o processo de gestação e o período pós-parto sejam permeados por medo e insegurança. ${ }^{14}$ As gestantes se sentem seguras com os encaminhamentos e informações concedidas pelos enfermeiros sobre a sua saúde geral e do bebê, o que aumenta a adesão nas atividades proposta durante o pré-natal.

Alguns fatores contribuem para a baixa representatividade do cirurgião-dentista aos serviços de prénatal odontológico onde a ausência da participação da odontologia em uma equipe interdisciplinar nesses serviços desperdiça uma excelente oportunidade de um encontro precoce entre dentista e gestante onde o profissional possa introduzir informações iniciais e incentivar a gestante quanto ao tratamento e prevenção da saúde bucal. ${ }^{15}$

Observa-se também o despreparo e a omissão de alguns profissionais odontólogos ao lidar com esse assunto, pois acabam postergando o atendimento às grávidas até o nascimento do bebê ao invés de sanar o problema odontológico ao ser diagnosticado, podendo ocasionar um dano maior em função do desenvolvimento da doença. ${ }^{16,5}$ Este fato revela a necessidade de investimentos em educação, tanto em nível de graduação como em pós-graduação, sobre a relação saúde bucal e gravidez para que os profissionais tenham maior segurança técnico-científica e torne-o um importante instrumento de promoção de saúde para essa população. ${ }^{17}$ 


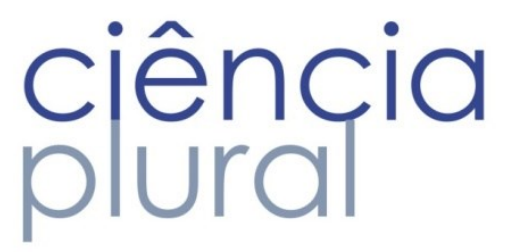

Têm-se que apenas que 15\% das gestantes confirmaram ter recebido esta orientação de seus médicos ginecologistas.16 Alguns resultados encontrados em pesquisas demonstraram que $81,9 \%$ dos médicos davam orientação quanto à saúde bucal às suas pacientes, porém apenas $8,2 \%$ indicaram a ida regular ao cirurgião-dentista e 21,3\% recomenda a visita somente quando as gestantes acharem necessário. ${ }^{18}$

Esses resultados ressaltam a necessidade de um intercâmbio de informações entre os profissionais de saúde para garantir um atendimento multiprofissional para desmistificar e orientar que atendimento odontológico pode ser realizado observando alguns cuidados: planejamento de sessões curtas, adequação da posição da cadeira, evitar consultas matinais e alguns procedimentos mais invasivos devem ser realizados no segundo trimestre da gestação.

Em outros estudos, parece consenso que a realização de intervenção em casos de urgência em qualquer período gestacional é mais benéfica para a mãe e o feto do que deixar a gestante sentindo estresse causado pela dor ou a disseminação de uma possível infecção. ${ }^{16,7}$

Ao se perguntar sobre a importância da ida ao dentista durante o período de gestação, todas as gestantes do presente estudo consideraram que era importante. Fica evidente em algumas falas que 0 período gestacional é um excelente momento para que o cirurgião-dentista seja um disseminador de informações e promova ações em saúde bucal com ênfase na promoção, prevenção de saúde da mãe e do bebê, podendo utilizar esses espaços para elucidar alguns fatos:5

"Sim, porque tipo, a gengiva sangra, a gente se desespera, tem enes motivos pra gente se desesperar por um fator que a gente não sabia que pode ocorrer na gestação." (G 03)

"É importante tanto pra mãe, como para o bebê." (G 01)

"Sim, pra prever todos esses problemas que causa na gestação." (G 07)

Os referidos resultados corroboram o fato de que a grande maioria das entrevistas com gestantes (90\%) considerou importante a visita ao dentista durante a gestação, sendo que os principais motivos relatados visaram somente à prevenção $(46 \%)$, à prevenção e ao tratamento curativo $(21 \%)$ e apenas ao tratamento curativo (23\%). ${ }^{16}$ Em outros estudos, a ida ao atendimento odontológico durante a gestação estava atrelada mediante autorização médica e durante atendimentos de emergência, e assim essa assistência odontológica seria postergada para depois do parto. ${ }^{7}$

O Cirurgião-dentista necessita se inserir em programas de pré-natal, vivenciando seu papel disseminador de informações a fim de modificar a condição de saúde bucal das mães. Espera-se então 


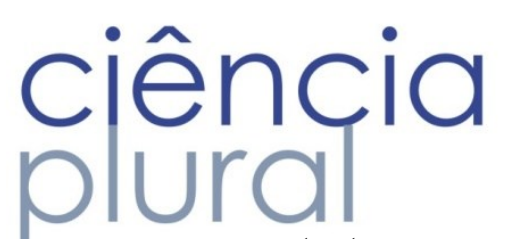

que as gestantes possam agir como agentes multiplicadores de saúde e todos os benefícios serão revertidos para seu bebê.

Concernente à ida das usuárias a uma consulta odontológica durante a gestação, 91,7\% disseram que compareceram, revelando que a maioria das usuárias referiu algum grau de adesão ao aconselhamento recebido, o que gera uma maior segurança e motivação para ir ao pré-natal odontológico. Isso é reflexo das melhorias assistenciais prestadas à gestante por meio de um esforço contínuo de todos os profissionais envolvidos nesse processo, de modo a garantir uma atenção integral a mulher, utilizando-se os meios existentes na comunidade e no ambiente de trabalho para facilitar ações promoção de saúde, melhoria da satisfação das usuárias, mediante um atendimento humanizado, eficaz, integral e igualitário. ${ }^{19,14}$

O vínculo entre familiares, gestantes e profissionais é um dos fundamentos importantes na abordagem odontológica para estabelecer confiança recíproca entre profissional e paciente, que acaba reduzindo o medo e o estresse nas consultas 1,20, facilitando o retorno da mãe ao consultório odontológico após o parto, para que o profissional possa contribuir nos cuidados com o bebê, em relação a hábitos de higiene oral, alimentação, e demais ações a serem desenvolvida principalmente pelo companheiro. ${ }^{20}$

Das gestantes entrevistadas, $8,3 \%$ das afirmaram não ter ido à consulta odontológica por não terem sido orientadas. É observado que alguns profissionais de saúde que lidam com maior frequência com as gestantes parecem não estar conscientes da importância da atenção odontológica no pré-natal e por isso deixam de contribuir, através de orientações e encaminhamentos para com a saúde das mulheres e de seus filhos, e/ou os profissionais não praticam esse cuidado de orientar durante uma consulta de rotina de pré-natal e a gestante deixa de ser beneficiada com tal orientação. 16,15

Como estratégia, os cirurgiões-dentistas podem utilizar a reunião de equipe como um instrumento relevante para melhorar o diálogo entre todos os profissionais da equipe de saúde, no intuito de facilitar e orientar sobre a importância de se fazer o acompanhamento do pré-natal odontológico, permitindo que nenhuma gestante deixe de ser assistida integralmente com seus cuidados.

Observou-se nesta pesquisa que $91,7 \%$ das gestantes acreditam que alteração na boca durante a gestação poderia influenciar na saúde geral do bebê, porém apenas uma minoria soube falar um pouco mais sobre o que poderia acontecer com seu filho:

"Sim, se você não tiver um acompanhamento e que a dentista explique a você sobre as doenças da boca pode influenciar na geração do bebe sim." (G 01) "Pode, pode causar parto prematuro." (G 06) 


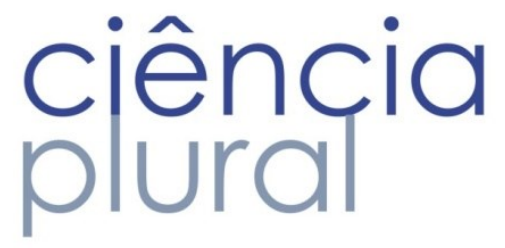

Algumas manifestações bucais são mais comuns durante a gestação como, por exemplo, a cárie e doença periodontal, embora a gravidez não seja a principal responsável por tais afecções. É sabido que mudanças hormonais podem diminuir o pH do meio bucal e a capacidade tampão da saliva, bem como a diminuição de cuidados com os hábitos alimentares nesse período, possíveis mudanças de hábitos de higiene bucal, além da frequência de ácidos provenientes de vômitos nesse período. Juntos todos estes fatores favorecem a atividade doença cárie. ${ }^{21,16}$

Durante a gestação, diversas infecções podem prejudicar o curso normal da gravidez chegando a comprometer a saúde do recém-nascido. A doença periodontal tem sido apontada como fator de risco para algumas intercorrências gestacionais, como parto pré-maturo e baixo peso ao nascer, porém alguns estudos do tipo revisões sistemáticas reportam uma possibilidade diminuída dessa associação com a doença periodontal, como também a tratamento periodontal não-cirúrgica aplicada rotineiramente em mulheres grávidas tráz a redução dos sinais da doença periodontal da mãe melhorando a sua saúde bucal. 22

É necessária a realização de estudos com metodologias mais rigorosas e com população diversa para a confirmação desta associação e da efetividade do tratamento periodontal na redução do risco destas intercorrências gestacionais. ${ }^{23,22}$

Considerando-se a ocorrência de diversas alterações durante o período gestacional, o profissional cirurgião-dentista precisa se empenhar em ações de cunho coletivo e atendimento individual a fim de informar às mães sobre todas as possíveis alterações que podem acontecer durante a gestação.

\section{Conclusão}

No presente estudo foram observados alguns aspectos positivos nas percepções do pré-natal odontológico pelas gestantes, tais como: as gestantes consideraram a importância do pré-natal odontológico, pois o viram como uma oportunidade e facilidade de acesso aos serviços odontológicos; também compareceram a consulta odontológica; apresentaram um grau de adesão ao aconselhamento fornecido pelos profissionais de saúde e também associaram que a presença de alguma alteração na boca poderia influenciar na saúde geral do bebê. Apesar dessas mudanças, foi observado que ainda é necessário estabelecer uma linguagem unificada entre os profissionais de saúde e uma maior conscientização do profissional Médico e Agente Comunitário de Saúde na orientação da gestante sobre o pré-natal odontológico, de modo que possam garantir a segurança do atendimento odontológico durante a gestação e implementar ações de promoção a saúde bucal trazendo benefícios de boas práticas de saúde tanto para mãe como para o futuro bebê. 


\section{ciência \\ plural}

\section{Referências}

1. Ministério da Saúde (BR), Secretaria de Atenção à Saúde. Departamento de Atenção Básica. Cadernos de Atenção Básica Atenção ao Pré-natal de Baixo Risco [Internet]. Brasília: Ministério da

Saúde; 2012 [cited 2017 may 28]. Available from: http://bvsms.saude.gov.br/bvs/publicacoes/cadernos_atencao_basica_32_prenatal.pdf

2. Pereira DS, Alves MB, Cavalcanti YW, Almeida-Marques RVD. Estudo dos fatores de risco à cárie dentária em gestantes conforme o trimestre gestacional. Rev bras ciênc saúde. 2012; 16(1): 29-34. Doi: 10.4034/RBCS.2012.16.01.05

3. Moimaz SAS, Carmo MP, Zina LG, Saliba NA. Associação entre condição periodontal de gestantes e variáveis maternas e de assistência à saúde. Pesqui bras odontopediatria clín integr. 2010; 10(2): 271-8. Doi: 10.4034/1519.0501.2010.0102.0021

4. Monteiro ACC, Pereira RM, Monteiro LPA, Costa ICC. Tratamento odontológico na gravidez: o que mudou na concepção das gestantes?. Revista Ciência Plural [Internet]. 2016 [cited 2017 may 30].; 2(2): 67-83. Available from: https://periodicos.ufrn.br/rcp/article/download/10903/7846.

5. Granville-Garcia AF, Leite AF, Smith LEA, Campos RVS, Menezes VA. Conhecimento de gestantes sobre saúde bucal no município de Caruaru-PE. Rev odontol UNESP [Internet]. 2007 [cited 2018 february 09]; 36(3): 243-9. Available from: http://s3.amazonaws.com/host-articleassets/rou/5880180c7f8c9d0a098b4a43/fulltext.pdf

6. Duarte KMM. Saúde da mulher: questões da prática assistencial para dentistas [Internet]. Universidade Aberta do SUS - UNA-SUS/UFMA. 2013 [cited 2017 may 07]; Available from: http://ares.unasus.gov.br/acervo/handle/ARES/549

7. Codato $L A B$, Nakama $L$, Melchior R. Percepções de gestantes sobre atenção odontológica durante a gravidez. Ciênc Saúde Colet. 2008; 13(3): 1075-80. Doi: 10.1590/S1413-81232008000300030

8. Konishi F. Odontologia intra-uterina. Rev Assoc Paul Cir Dent. 1995 Mar./Abr.; 49(2): 135-6.

9. Toledo MTT, Abreu MN, Lopes ACS. Adesão a modos saudáveis de vida mediante aconselhamento por profissionais de saúde. Revista Saúde Pública. 2013; 47(3): 540-8. Doi: 10.1590/S00348910.2013047003936

10. MINAYO MCS. O desafio do conhecimento. Pesquisa qualitativa em saúde. ga edição revista e aprimorada. São Paulo: Hucitec, 2006. Doi: 10.1590/S1413-81232007000400030 


\section{ciência plural}

11. Fontanella BJB, Ricas J, Turato ER. Amostragem por Saturação em Pesquisa Qualitativa em Saúde: Contribuições Teóricas. Cadernos de Saúde Pública 2008; 24(1): 17-27. Doi: 10.1590/S0102$311 \times 2008000100003$

12. Campos L, Bottan ER, Birolo JB, Silveira EG, Schmitt BHE. Conhecimento de mães de diferentes classes sociais sobre saúde bucal no município de Cocal do Sul (SC) [Internet]. RSBO. 2010 [cited 2017 may 13]; 7(3): 287-95. Available from: http://univille.edu.br/account/odonto/VirtualDisk.html?action=readFile\&file=Artigo_05.pdf\&current=/R SBO_-_v.7_-_n.03-_julho-setembro_2010

13. Lawder JAC, Mendes YBE, Silva LC, Andrade DKD, Rocha LM, Rogalla TM, et al. Conhecimento e práticas em saúde bucal entre usuários de serviços odontológicos. Pesqui Bras Odontopediatria Clín Integr. 2009; 8(3): 321-6. Doi: 10.4034/1519.0501.2008.0083.0011

14. Guerreiro EM, Rodrigues DP, Silveira MAM, Lucena NBF. O cuidado pré-natal na atenção básica de saúde sob o olhar de gestantes e enfermeiros. REME. 2012; 16(3): 315-23. Doi: 10.1590/S141527622012000300002

15. Finkler M, Oleiniski DMB, Ramos FRS. Saúde bucal materno-infantil: um estudo de representações sociais com gestantes. Texto Contexto - enferm. 2004; 13(3): 360-8. Doi: 10.1590/S010407072004000300004

16. Bastiane C, Cota ALS, Provenzano MGA, Fracasso MLC, Honório HM, Rios D. Conhecimento das gestantes sobre alterações bucais e tratamento odontológico durante a gravidez. Odontol Clín - Cient. 2010; 9(2): 155-60. Doi: S1677-38882010000200013

17. Codato LAB, Nakama L, Júnior LC, Higasi MS. Atenção odontológica à gestante: papel dos profissionais de saúde. Ciênc Saúde Colet. 2011; 16(4): 2297-2301. Doi: 10.1590/S141381232011000400029

18. Menoli APV, Frossard WTG. Perfil de médicos ginecologistas/obstetras de Londrina com relação à saúde oral da gestante. Semina Ciênc Biol Saúde. 2004; 18(1): 34-42. Doi: 10.5433/16790367.1997v18n1p34

19. Ministério da Saúde (Brasil). Secretaria de Atenção à Saúde. Departamento de Ações Programáticas Estratégicas. Política Nacional de Atenção Integral à Saúde da Mulher. Plano de Ação 2004-2007 [Internet]. Brasília: Ministério da Saúde. 2004 [cited 2017 may 17]; Available from: http://bvsms.saude.gov.br/bvs/publicacoes/politica_nac_atencao_mulher2.pdf

20. Mameluque S, Souza Júnior EB, Rezende JC, Costa CCG, Vanham IM, Oliveira JM, et al. Abordagem integral no atendimento odontológico à gestante [Internet]. Rev Unimontes Científica. 2005 [cited 2017 


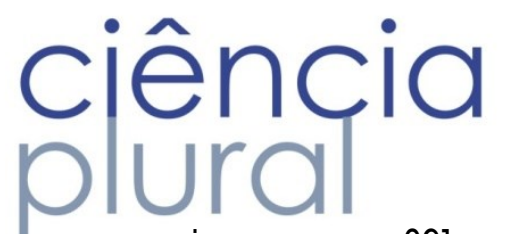

june

09];

Jan./Jun.

$7(1)$ :

67-76.

Available

from:

http://www.ruc.unimontes.br/index.php/unicientifica/article/viewFile/81/75

21. Moimaz SAS, Rocha NB, Saliba O, Garbin CAS. O acesso de gestantes ao tratamento odontológico. Rev Odontol da Univ Cid de São Paulo. 2007 [cited 2017 may 17]; 19(1): 39-45. Available from: http://arquivos.cruzeirodosuleducacional.edu.br/principal/old/revista_odontologia/pdf/3_janeiro_abril_ 2007/0_acesso_gestantes.pdf

22. Yassin García SA, Alonso Rosado A, García López M, García Moreno ME, Pérez Gómez RM, Bascones Martínez A. Enfermedad periodontal y resultados adversos del embarazo: revisión de la literatura. Part 2. Avances en Periodoncia Implantología Oral [Internet]. 2016 [cited 2018 february 15]; 28(3): 137-45. Available from: http://scielo.isciii.es/scielo.php?script=sci_isoref\&pid=S1699$65852016000300003 \&$ Ing=es\&tlng=e

23. Costa LCM, Amormino SAF, Albuquerque BN, Cota LOM, Costa JED, Costa FO. Doença periodontal e intercorrências gestacionais. Perionews. 2012 [cited 2018 february 15]; 6(1): 61-5. Available from: http://www.inpn.com.br/InPerio/Artigo/Index/914\#

Submetido: $10 / 08 / 2018$

Aprovado: 31/12/2018 